REPRESENTATION THEORY

An Electronic Journal of the American Mathematical Society

Volume 10, Pages 130-146 (February 20, 2006)

S $1088-4165(06) 00200-7$

\title{
PURITY OF EQUIVALUED AFFINE SPRINGER FIBERS
}

\author{
MARK GORESKY, ROBERT KOTTWITZ, AND ROBERT MACPHERSON
}

\begin{abstract}
The affine Springer fiber corresponding to a regular integral equivalued semisimple element admits a paving by vector bundles over Hessenberg varieties and hence its homology is "pure".
\end{abstract}

\section{INTRODUCTION}

1.1. Overview. In GKM04 the authors developed a geometric approach to the study of the "kappa" orbital integrals $\mathcal{O}_{u}^{\kappa}\left(1_{\mathfrak{k}}\right)$ that arise in the conjecture L83] $\S$ III.1 of R. Langlands that is commonly referred to as the "fundamental lemma". Theorem 15.8 of GKM04 expresses $\mathcal{O}_{u}^{\kappa}\left(1_{\mathfrak{k}}\right)$ as the Grothendieck-Lefschetz trace of the action of Frobenius on the étale cohomology of the quotient $\Lambda \backslash X_{u}$ of a certain affine Springer fiber $X_{u}$ by its lattice $\Lambda$ of translations. (Precise definitions will follow below.) The authors also developed a procedure which, under three assumptions, gives an explicit formula for the étale cohomology of $\Lambda \backslash X_{u}$. The three assumptions were:

(1) the element $u$ lies in an unramified torus,

(2) the étale homology of $X_{u}$ is pure,

(3) equivariant étale homology satisfies certain formal properties analogous to those of equivariant singular homology.

Assumption (2) was referred to as the "purity hypothesis" and it has also been adopted by G. Laumon Lau in a recent preprint.

Under hypothesis (1) (the "unramified case"), a certain split torus $S$ acts on the affine Springer fiber $X_{u}$. The purity assumption (2) implies that the homology of $X_{u}$ is equivariantly formal with respect to the action of $S$. Corollary 7.5 of GKM98 then provides an expression for the equivariant homology in terms of the 0 and 1 dimensional orbits of $S$. This is the main technical tool in the geometric approach of (GKM04 §15.12) to the orbital integral $\mathcal{O}_{u}^{\kappa}\left(1_{\mathfrak{k}}\right)$. Thus, the purity hypothesis is an essential ingredient in this approach, and in fact the authors conjectured (cf. GKM04 Conjecture 5.3) that the homology of the affine Springer fiber $X_{u}$ is pure, whether or not the element $u$ lies in an unramified torus.

Purity is often a consequence of (a) nonsingularity or (b) the existence of a paving by affine spaces. Both $X_{u}$ and $\Lambda \backslash X_{u}$ are highly singular (in general), and

Received by the editors July 3, 2003 and, in revised form, October 19, 2005.

2000 Mathematics Subject Classification. Primary 22E67; Secondary 22E35.

The research of M. G. was supported in part by N. S. F. grant DMS-0139986 and DARPA grant HR0011-04-1-0031.

The research of R. K. was supported in part by N. S. F. grants DMS-0071971 and DMS-0245639.

(C)2006 American Mathematical Society 
they may have cohomology in odd degrees, so neither of these techniques can be used.

In this paper we verify the purity assumption (2) for elements $u$ that are "equivalued", but not necessarily unramified. We show that $X_{u}$ has a paving by iterated affine space bundles over certain (nonsingular) Hessenberg varieties, and this implies purity. In fact, we show that such "Hessenberg pavings" exist, not just for the affine Springer fibers of GKM04, but for a more general class of schemes that we refer to as "generalized affine Springer fibers". The proof comprises most of this paper and it concludes in Section 5.5. but for the convenience of the reader it is summarized in Section 7 . We now make the above concepts and statements more precise.

1.2. Let $k$ be an algebraically closed field, $G$ a connected reductive group over $k$, and $A$ a maximal torus in $G$. We write $\mathfrak{g}$ for the Lie algebra of $G$ and $\mathfrak{a}$ for $X_{*}(A) \otimes \mathbf{z} \mathbf{R}$. Let $F=k((\epsilon))$ be the field of formal Laurent series over $k$ and let $\mathfrak{o}=k[[\epsilon]]$ be the subring of formal power series. We fix an algebraic closure $\bar{F}$ of $F$. We write $\mathbf{G}$ and $\mathfrak{g}$ for $G(F)$ and $\mathfrak{g}(F):=\mathfrak{g} \otimes_{k} F$ respectively. Let $y \in \mathfrak{a}$ and write $\mathbf{G}_{y}$ and $\mathfrak{g}_{y}$ for the associated (connected) parahoric subgroup and subalgebra respectively. For example, if $y=0$, then $\mathbf{G}_{y}=G(\mathfrak{o})$ and $\mathfrak{g}_{y}=\mathfrak{g}(\mathfrak{o})$, while if $y$ lies in the interior of an alcove, then $\mathbf{G}_{y}$ (resp., $\mathfrak{g}_{y}$ ) is an Iwahori subgroup (resp. subalgebra). We write $\mathcal{F}_{y}$ for the $k$-ind-scheme $\mathbf{G} / \mathbf{G}_{y}$. When $y=0$, for example, $\mathcal{F}_{y}$ is the affine Grassmannian $G(F) / G(\mathfrak{o})$. Any $u \in \mathfrak{g}$ determines a closed subset

$$
\mathcal{F}_{y}(u):=\left\{g \in \mathbf{G} / \mathbf{G}_{y}: \operatorname{Ad}\left(g^{-1}\right)(u) \in \mathfrak{g}_{y}\right\}
$$

of $\mathcal{F}_{y}$, called an affine Springer fiber. Affine Springer fibers were first studied by Kazhdan and Lusztig [KL88, who established some of their basic properties, one of which we will now recall. Let $T$ be a maximal $F$-torus in $G$, let $\mathfrak{t}$ be its Lie algebra (over $F$ ), and let $A_{T}$ denote the maximal $F$-split torus in $T$. A regular element $u \in \mathfrak{t}$ is said to be integral if val $\lambda(u) \geq 0$ for every $\lambda \in X^{*}(T)$ (convention: val $0=+\infty)$. Here we have denoted the value of the differential of $\lambda$ on $u$ simply by $\lambda(u)$, an especially abusive notation when $k$ has characteristic $p$, since in that case the differential of $\lambda$ is 0 whenever $\lambda$ is divisible by $p$ in $X^{*}(T)$. The centralizer $T(F)$ of $u$ acts on $\mathcal{F}_{y}(u)$, and inside the centralizer we have the lattice $\Lambda:=X_{*}\left(A_{T}\right)$, viewed as a subgroup of $T(F)$ by sending $\mu \in \Lambda$ to $\epsilon^{\mu} \in T(F)$. The lattice $\Lambda$ acts freely on $\mathcal{F}_{y}(u)$, and Kazhdan and Lusztig showed that the quotient $\Lambda \backslash \mathcal{F}_{y}(u)$ is a projective (usually singular) variety over $k$, non-empty if and only if $u$ is integral; and, for tamely ramified $T$, they conjectured a formula for its dimension, later proved by Bezrukavnikov Bez96].

In order to state the main result of this paper, we need two more definitions. We normalize the valuation on $\bar{F}$ so that val $\epsilon=1$. We say that regular $u \in \mathfrak{t}$ is equivalued with valuation $s \in \mathbf{Q}$ if $\operatorname{val} \alpha(u)=s$ for every root $\alpha$ of $T$ over $\bar{F}$ and $\operatorname{val} \lambda(u) \geq s$ for every $\lambda \in X^{*}(T)$. (For adjoint groups $G$ the second part of the condition is of course redundant.) When $s$ plays no role, we say simply that $u$ is equivalued.

Hessenberg varieties were introduced by De Mari, Procesi and Shayman MPS92. In $\S 1$ below we define them in greater generality but still call them Hessenberg varieties. The ones in MPS92 are non-empty and admit pavings by affine spaces, but this is not always true of the more general ones considered below (see 2.6 for the emptiness question). In any case Hessenberg varieties are certain very special non-singular closed subvarieties of (partial) flag manifolds. By a Hessenberg 
paving of an ind-scheme $X$ over $k$ we mean an exhaustive increasing filtration $X_{0} \subset X_{1} \subset X_{2} \subset \ldots$ of $X$ by closed complete subschemes $X_{i}$ of finite type over $k$ such that each successive difference $X_{i} \backslash X_{i-1}$ is a disjoint union of iterated affine space bundles over Hessenberg varieties.

Theorem 1.1. Assume that the order of the absolute Weyl group $W$ of $G$ is invertible in $k$. Let $u$ be a regular integral equivalued element of $\mathfrak{t}$. Then the affine Springer fiber $\mathcal{F}_{y}(u)$ admits a Hessenberg paving.

For some tori $T$ all the (non-empty) Hessenberg varieties turn out to be single points, in which case the Hessenberg paving becomes a paving by affine spaces. Before stating this as a precise theorem, we need to recall that (assuming $|W|$ is invertible in $k$ ) the $G(F)$-conjugacy classes of maximal $F$-tori in $G$ are parametrized by conjugacy classes in $W$. (This well-known fact is reviewed in [5.2.) We say that $T$ is Coxeter if the associated conjugacy class in $W$ consists of Coxeter elements. Now consider the centralizer $M$ of $A_{T}$ in $G$. Then $M$ is a Levi subgroup of $G$ (hence is split over $F$ ) and contains $T$ as an elliptic maximal $F$-torus. We say that $T$ is weakly Coxeter in $G$ if $T$ is Coxeter in $M$. For example, split maximal tori are weakly Coxeter, and all maximal tori in $G L(n)$ are weakly Coxeter.

Our Hessenberg pavings are obtained by intersecting the affine Springer fiber with the orbits of a parahoric subgroup that depends on $T$. In 4.8 we give a formula for the dimensions of these intersections. When the parahoric subgroup turns out to be an Iwahori subgroup, for example, when $T$ is weakly Coxeter, we get a paving by affine spaces, reminiscent of the one found by De Concini, Lusztig and Procesi CLP88 for ordinary (that is, non-affine) Springer fibers, and coinciding with the one found by Lusztig and Smelt LS91] in the case of homogeneous $u \in \mathfrak{t}$, with $T$ a Coxeter torus in $G L(n)$. More precisely, we have the following theorem, in which the dimensions of the affine spaces mentioned in its statement can be determined using 4.8

Theorem 1.2. Assume that $|W|$ is invertible in $k$ and that $T$ is weakly Coxeter. Let $u$ be a regular integral equivalued element of $\mathfrak{t}$. Then the affine Springer fiber $\mathcal{F}_{y}(u)$ admits a paving by affine spaces.

It is worth noting that the equivalued hypothesis used here is weaker than the customary one of homogeneity. However, the homogeneity hypothesis has the virtue of guaranteeing the existence of a useful $\mathbf{G}_{m}$-action on the affine Springer fiber, stemming from the automorphisms $\epsilon \mapsto z \epsilon$ of $F\left(z \in \mathbf{G}_{m}\right)$. The work of Lusztig and Smelt has been generalized to homogeneous elements in $\mathfrak{t}$ for Coxeter tori $T$ by Fan [Fan96, Sage Sag00 and Sommers Som97. Quite recently these results have been reconsidered in the context of Cherednik's double-affine Hecke algebras by Vasserot Vas and by Berest, Etingof and Ginzburg BEG. A homogeneous (weakly Coxeter) case for $G L(n)$ was analyzed by Laumon and Waldspurger [LW].

It is also worth noting that Hessenberg pavings which are not pavings by affines are plentiful for groups other than $G L(n)$. Indeed this occurs in the example of Bernstein and Kazhdan, discussed in the appendix to KL88, in which one of the irreducible components of the affine Springer fiber for a certain kind of homogeneous element in $\mathfrak{s p}(6)$ admits a dominant map to an elliptic curve.

In the body of the paper we actually allow a more general set-up, with the role of $u \in \mathfrak{g}$ being played by vectors $v \in \mathbf{V}:=V \otimes_{k} F$, where $V$ is a finite dimensional representation of $G$ over $k$, and with the role of $\mathfrak{g}_{y}$ being played by lattices $\mathbf{V}_{y, t}$ in $\mathbf{V}$ 
$(t \in \mathbf{R})$ analogous to the Moy-Prasad [MP94] lattices $\mathfrak{g}_{y, t}$ in $\mathfrak{g}$. In this situation there are (generalized) affine Springer fibers $\mathcal{F}_{y}(t, v)$ (see 4.1), having Hessenberg pavings under suitable hypotheses on $t$ and $v$ (see 4.2).

Thus, even in the traditional case $V=\mathfrak{g}$, in the body of the paper the parahoric subalgebra $\mathfrak{g}_{y}$ occurring in the definition of $\mathcal{F}_{y}(u)$ is replaced by a Moy-Prasad lattice $\mathfrak{g}_{y, t}$, and we construct a Hessenberg paving of the (generalized) affine Springer fiber $\mathcal{F}_{y}(t, u)$ for any equivalued $u \in \mathfrak{t}$ with valuation $s \geq t$, again under the assumption that $|W|$ is invertible in $k$. The special case $t=0$ yields the theorem stated earlier.

Theorem 1.1 of this paper yields purity results used to study orbital integrals in GKM04 and in a recent preprint of Laumon Lau. Indeed, suppose that $G$ is defined and split over a finite subfield $k_{0}$ of $k$, and that $T$ and $u$ are defined over $k_{0}((\epsilon))$. Then our Hessenberg pavings are defined over some finite extension of $k_{0}$ in $k$. The (easy) proof of the following corollary can be found in Section 7.2 ,

Corollary 1.3. Suppose that $u \in \mathfrak{t}$ is equivalued with valuation $s \geq t$. Assume $|W|$ is invertible in $k$ and that $l$ is relatively prime to the characteristic of $k$. Then all eigenvalues of the geometric Frobenius acting on the étale homology $H_{i}\left(\mathcal{F}_{y}(t, u), \mathbf{Q}_{l}\right)$ have complex absolute value $q^{i / 2}$.

1.3. We wish to draw attention to the paper, Luc03] of V. Lucarelli, who shows that certain affine Springer fibers for PGL(3) with unequal valuation admit pavings by affines, thereby establishing purity in those cases. We would like to thank an anonymous referee for useful suggestions.

\section{Hessenberg VARIEties}

Throughout this section we consider finite dimensional representations $V$ of $G$. For $\lambda \in X^{*}(A)$ let $V_{\lambda}$ denote the weight space $\{v \in V: a v=\lambda(a) v$ for all $a \in A\}$.

2.1. Filtrations on representations $V$ of $G$. Let $y \in \mathfrak{a}$. As usual $y$ gives rise to an $\mathbf{R}$-grading on $V$ :

$$
V=\bigoplus_{t \in \mathbf{R}} V(y, t)
$$

where $V(y, t)$ is the direct sum of the weight spaces $V_{\lambda}$ for weights $\lambda \in X^{*}(A)$ such that $\lambda(y)=t$. We will also need the associated filtrations $F_{y}^{t}$ on $V$, where the subspace $F_{y}^{t} V$ is by definition $\bigoplus_{t^{\prime} \geq t} V\left(y, t^{\prime}\right)$. Clearly, $F_{y}^{t} V \subset F_{y}^{s} V$ if $s \leq t$.

2.2. Unstable vectors. As usual in geometric invariant theory we say that a vector $v \in V$ is $G$-unstable if there exist $g \in G, y \in \mathfrak{a}$ and $t>0$ such that $v \in g \cdot F_{y}^{t} V$.

2.3. Good vectors. We say that a vector $v \in V$ is $G$-good if there is no nonzero $G$-unstable vector $v^{*} \in V^{*}$ that vanishes identically on the subspace $\mathfrak{g} \cdot v$ of $V$. (Here $V^{*}$ denotes the vector space dual to $V$.) For example, any regular semisimple element in $\mathfrak{g}$ is $G$-good, at least if the characteristic of $k$ is different from 2 (see 6.5).

2.4. Partial flag manifold $\mathcal{P}_{y}$. Let $y \in \mathfrak{a}$. As usual $y$ determines a parabolic subgroup $P_{y}$ of $G$ whose Lie algebra is $F_{y}^{0} \mathfrak{g}$. We write $\mathcal{P}_{y}$ for the homogeneous space $G / P_{y}$. The subspaces $F_{y}^{t} V$ are $P_{y}$-stable, so there is a $G$-equivariant vector bundle $E_{y}^{t} V$ over $\mathcal{P}_{y}$ whose fiber at $g P_{y} \in \mathcal{P}_{y}$ is equal to the subspace $g F_{y}^{t} V$ of $V$. 
2.5. Hessenberg varieties. The vector bundle $E_{y}^{t} V$ is a subbundle of the constant vector bundle $\tilde{V}$ over $\mathcal{P}_{y}$ with fiber $V$, so we may consider the quotient vector bundle $\tilde{V} / E_{y}^{t} V$ on $\mathcal{P}_{y}$. Any vector $v \in V$ determines a global section of $\tilde{V} / E_{y}^{t} V$, and we define $\mathcal{P}_{y}(t, v)$ to be the zero-set of this global section, a closed subscheme of $\mathcal{P}_{y}$. Thus $\mathcal{P}_{y}(t, v)=\left\{g \in G / P_{y}: g^{-1} v \in F_{y}^{t} V\right\}$.

The map $Y \mapsto Y \cdot v$ from $\mathfrak{g}$ to $V$ can be viewed as a constant map $\cdot v: \tilde{\mathfrak{g}} \rightarrow \tilde{V}$ of constant vector bundles. Over $\mathcal{P}_{y}(t, v)$ the vector $v$ lies in $E_{y}^{t} V$, so the map $\cdot v$ carries $E_{y}^{0} \mathfrak{g}$ into $E_{y}^{t} V$, yielding an induced map

$$
\cdot v: \tilde{\mathfrak{g}} / E_{y}^{0} \mathfrak{g} \rightarrow \tilde{V} / E_{y}^{t} V
$$

The tangent bundle to $\mathcal{P}_{y}$ is $\tilde{\mathfrak{g}} / E_{y}^{0} \mathfrak{g}$, and it is easy to see that the Zariski tangent space to $\mathcal{P}_{y}(t, v)$ at a point of $\mathcal{P}_{y}(t, v)$ is equal to the kernel of $\cdot v: \tilde{\mathfrak{g}} / E_{y}^{0} \mathfrak{g} \rightarrow \tilde{V} / E_{y}^{t} V$ on the fibers at that point.

Now suppose that $v$ is a $G$-good vector and that $t \leq 0$, in which case we refer to $\mathcal{P}_{y}(t, v)$ as a Hessenberg variety. We claim that any Hessenberg variety is nonsingular and projective. Projectivity is clear. To prove non-singularity we will check that the global section $v$ of $\tilde{V} / E_{y}^{t} V$ is transverse to the zero-section, or in other words that the map $\cdot v: \tilde{\mathfrak{g}} / E_{y}^{0} \mathfrak{g} \rightarrow \tilde{V} / E_{y}^{t} V$ is surjective on fibers at each point $g P_{y} \in \mathcal{P}_{y}(t, v)$. To prove surjectivity at this point we must show that any $v^{*} \in V^{*}$ that annihilates both $\mathfrak{g} \cdot v$ and $g \cdot F_{y}^{t} V$ is zero. Since $v^{*}$ annihilates the second subspace and $t \leq 0$, there exists $\delta>0$ such that $v^{*} \in g \cdot F_{y}^{\delta} V^{*}$, and therefore $v^{*}$ is $G$-unstable. Since $v$ is $G$-good, it follows that $v^{*}=0$.

2.6. When are Hessenberg varieties empty? Hessenberg varieties are sometimes empty. Fortunately, there is a fairly practical way to determine when this happens, as we will now see. With notation as above, choose a Borel subgroup $B$ of $G$ containing $A$ and contained in $P_{y}$. The closed subvariety

$$
Y:=\left\{g \in G / B: g^{-1} v \in F_{y}^{t} V\right\}
$$

of the flag manifold $G / B$ obviously maps onto the Hessenberg variety $\mathcal{P}_{y}(t, v)$, and therefore it is enough to determine when $Y$ is empty.

Now $Y$ is empty precisely when its fundamental class in $H^{\bullet}\left(G / B, \mathbf{Q}_{l}\right.$ ) is 0 (where $l$ is a prime that is non-zero in our ground field $k$ ). Recall that the $\mathbf{Q}_{l}$-algebra $H^{\bullet}\left(G / B, \mathbf{Q}_{l}\right)$ is the quotient of the symmetric algebra $S^{\bullet}$ on $X^{*}(A) \otimes \mathbf{z} \mathbf{Q}_{l}$ by the ideal $I$ generated by the Weyl group invariant elements that are homogeneous of strictly positive degree.

It follows from the discussion in 2.5 that $Y$ is obtained as the zero-set of a global section of the $G$-equivariant vector bundle on $G / B$ obtained from the representation $V / F_{y}^{t} V$ of $B$, and that, moreover, this global section is transverse to the zero-section. Therefore the fundamental class of $Y$ in $H^{\bullet}\left(G / B, \mathbf{Q}_{l}\right)$ is equal to the top Chern class of our vector bundle on $G / B$. Filtering $V / F_{y}^{t} V$ by a complete flag of $B$-stable subspaces, we see that our top Chern class is the image under $S^{\bullet} \rightarrow H^{\bullet}\left(G / B, \mathbf{Q}_{l}\right)$ of the element $\lambda_{1} \ldots \lambda_{m}$, where $m$ is the dimension of $V / F_{y}^{t} V$ and $\lambda_{1}, \ldots, \lambda_{m}$ are the characters of $A$ (with multiplicities) occurring in the $B$-module $V / F_{y}^{t} V$.

We conclude that our Hessenberg variety is empty if and only if $\lambda_{1} \ldots \lambda_{m}$ lies in the ideal $I$. 
2.7. Some vector bundles over Hessenberg varieties in the graded case. The following constructions will be used to produce the Hessenberg pavings of Section 3.

Let $x \in \mathfrak{a}$. We use $x$ to define an $\mathbf{R} / \mathbf{Z}$-grading,

$$
V=\bigoplus_{s \in \mathbf{R} / \mathbf{Z}} V(x, s+\mathbf{Z})
$$

on $V$, where

$$
V(x, s+\mathbf{Z}):=\bigoplus_{m \in \mathbf{Z}} V(x, s-m),
$$

with $V(x, s-m)$ as in 2.1. In this way $\mathfrak{g}$ itself becomes an $\mathbf{R} / \mathbf{Z}$-graded Lie algebra and $V$ becomes an $\mathbf{R} / \mathbf{Z}$-graded $\mathfrak{g}$-module.

We denote by $H$ the subgroup of $G$ generated by $A$ and the root subgroups for all roots $\alpha$ of $A$ in $\mathfrak{g}$ such that $\alpha(x) \in \mathbf{Z}$. Then $H$ is a connected reductive group whose Lie algebra is $\mathfrak{g}(x, 0+\mathbf{Z})$. The action of $H$ on $V$ preserves the $\mathbf{R} / \mathbf{Z}$-grading of $V$.

Now let $y \in \mathfrak{a}$ and $t \in \mathbf{R}$. Since $A$ is also a maximal torus in $H$, we may apply the construction in 2.1 to the representation $V(x, \mathbf{s})$ of $H$, where we have written $\mathbf{s}$ as an abbreviation for $s+\mathbf{Z}$. In this way we get a vector subbundle $E_{y}^{t} V(x, \mathbf{s})$ of the constant vector bundle $\tilde{V}(x, \mathbf{s})$ on $\mathcal{P}_{y}=H / P_{y}$.

Let us fix $x, y \in \mathfrak{a}, s, t \in \mathbf{R}$ and $v \in V(x, \mathbf{s})$. We assume further that $t \leq 0$ and that the image of $v$ in $V$ is a $G$-good vector (which implies that $v$ is $H$-good in $V(x, \mathbf{s})$ ). Under these hypotheses we are going to define some vector bundles over the Hessenberg variety $\mathcal{P}_{y}(t, v) \subset \mathcal{P}_{y}=H / P_{y}$ associated to the vector $v$ in the representation $V(x, \mathbf{s})$ of $H$.

The vector bundles depend on two additional real numbers $r$ and $t^{\prime}$. We write $\mathbf{r}$ for $r+\mathbf{Z}$, and we put $t^{\prime \prime}:=t+t^{\prime}$. We assume that $t^{\prime \prime} \leq 0$. The action of $\mathfrak{g}$ on $V$ induces a linear map

$$
\mathfrak{g}(x, \mathbf{r}) \otimes V(x, \mathbf{s}) \rightarrow V(x, \mathbf{r}+\mathbf{s}) .
$$

This gives rise to a constant homomorphism

$$
\tilde{\mathfrak{g}}(x, \mathbf{r}) \otimes \tilde{V}(x, \mathbf{s}) \rightarrow \tilde{V}(x, \mathbf{r}+\mathbf{s})
$$

of constant vector bundles which carries $E_{y}^{t^{\prime}} \mathfrak{g}(x, \mathbf{r}) \otimes E_{y}^{t} V(x, \mathbf{s})$ into $E_{y}^{t^{\prime \prime}} V(x, \mathbf{r}+\mathbf{s})$. Over the Hessenberg variety $\mathcal{P}_{y}(t, v)$ the global section $v$ lies in the subbundle $E_{y}^{t} V(x, \mathbf{s})$. Therefore the map $Y \mapsto Y \cdot v$ induces a homomorphism

$$
\cdot v: \tilde{\mathfrak{g}}(x, \mathbf{r}) / E_{y}^{t^{\prime}} \mathfrak{g}(x, \mathbf{r}) \rightarrow \tilde{V}(x, \mathbf{r}+\mathbf{s}) / E_{y}^{t^{\prime \prime}} V(x, \mathbf{r}+\mathbf{s}),
$$

and in fact this homomorphism is surjective at every point of the Hessenberg variety, as one sees using the $G$-goodness of $v$ and the fact that $t^{\prime \prime} \leq 0$, just as in the proof of non-singularity of Hessenberg varieties. It follows that the kernel of the homomorphism, call it $K\left(x, y, r, s, t, t^{\prime}, v\right)$, is a vector bundle. Denoting by $H_{v}$ the stabilizer of $v$ in $H$ (which of course acts on our Hessenberg variety), we have an exact sequence of $H_{v}$-equivariant vector bundles on the Hessenberg variety $\mathcal{P}_{y}(t, v)$ :

$$
0 \rightarrow K\left(x, y, r, s, t, t^{\prime}, v\right) \rightarrow \tilde{\mathfrak{g}}(x, \mathbf{r}) / E_{y}^{t^{\prime}} \mathfrak{g}(x, \mathbf{r}) \rightarrow \tilde{V}(x, \mathbf{r}+\mathbf{s}) / E_{y}^{t^{\prime \prime}} V(x, \mathbf{r}+\mathbf{s}) \rightarrow 0 .
$$




\section{MOY-PRASAD GRADINGS AND FILTRATIONS}

3.1. Moy-Prasad filtrations on representations $V$ of $G$. As in the introduction we write $\mathbf{V}$ for $V \otimes_{k} F$ and $\mathfrak{g}$ for $\mathfrak{g} \otimes_{k} F$, where $F=k((\epsilon))$. The subspace $V \otimes_{k} k\left[\epsilon, \epsilon^{-1}\right]$ of $\mathbf{V}$ can be written as a direct sum of the subspaces $V_{\lambda} \epsilon^{m}$, where $(\lambda, m)$ ranges through $X^{*}(A) \oplus \mathbf{Z}$.

Now suppose that $x \in \mathfrak{a}$. For $r \in \mathbf{R}$ we define a subspace $\mathbf{V}_{x}(r)$ of $\mathbf{V}$ by

$$
\mathbf{V}_{x}(r)=\bigoplus_{\lambda(x)+m=r} V_{\lambda} \epsilon^{m}
$$

(In other words, the direct sum is taken over all $(\lambda, m) \in X^{*}(A) \oplus \mathbf{Z}$ such that $\lambda(x)+m=r$.) Thus we have

$$
V \otimes_{k} k\left[\epsilon, \epsilon^{-1}\right]=\bigoplus_{r \in \mathbf{R}} \mathbf{V}_{x}(r) .
$$

Now put $\mathbf{V}_{x, r}:=\prod_{r^{\prime} \geq r} \mathbf{V}_{x}\left(r^{\prime}\right)$. Clearly, $\mathbf{V}_{x, r} \subset \mathbf{V}_{x, s}$ if $r \geq s$, so we have defined an R-filtration on $\mathbf{V}$. Put $\mathbf{V}_{x, r+}:=\prod_{r^{\prime}>r} \mathbf{V}_{x}\left(r^{\prime}\right)$. Thus we have exact sequences

$$
0 \rightarrow \mathbf{V}_{x, r+} \rightarrow \mathbf{V}_{x, r} \rightarrow \mathbf{V}_{x}(r) \rightarrow 0 \text {. }
$$

Clearly, $\mathbf{V}_{x, r+}=\mathbf{V}_{x, r+\delta}$ for all sufficiently small $\delta>0$.

Taking $V=\mathfrak{g}$, we get $\mathfrak{g}_{x}(r)$ and $\mathfrak{g}_{x, r}$. Note that $\mathfrak{g}_{x, r}$ are the lattices in $\mathfrak{g}$ defined by Moy-Prasad MP94. In this way $\mathfrak{g}$ becomes an $\mathbf{R}$-filtered Lie algebra over $k$ and $\mathbf{V}$ an $\mathbf{R}$-filtered $\mathfrak{g}$-module. In other words, the action of $\mathfrak{g}$ on $\mathbf{V}$ induces linear maps

and

$$
\mathfrak{g}_{x, r} \otimes \mathbf{V}_{x, s} \rightarrow \mathbf{V}_{x, r+s}
$$

$$
\mathfrak{g}_{x}(r) \otimes \mathbf{V}_{x}(s) \rightarrow \mathbf{V}_{x}(r+s) .
$$

Recall the subspace $V(x, \mathbf{r})$ of $V$ defined in 2.7. where $\mathbf{r}$ denotes $r+\mathbf{Z}$, as before. We are now going to define a canonical linear isomorphism

$$
i_{x, r}: \mathbf{V}_{x}(r) \rightarrow V(x, \mathbf{r}) .
$$

The space $\mathbf{V}_{x}(r)$ is the direct sum of the spaces $V_{\lambda} \epsilon^{m}$ for $\lambda, m$ such that $\lambda(x)+m=$ $r$. For such $\lambda, m$ define a linear isomorphism $V_{\lambda} \epsilon^{m} \rightarrow V_{\lambda}$ by sending $v \epsilon^{m}$ to $v$. The direct sum of these isomorphisms is the desired isomorphism $\mathbf{V}_{x}(r) \rightarrow V(x, \mathbf{r})$. Under these isomorphisms the linear map (3.1.1) goes over to the linear map (2.7.1).

3.2. Moy-Prasad subgroups of G. We denote $G(F)$ by $\mathbf{G}$. For $x \in \mathfrak{a}$ and $r \geq 0$ Moy-Prasad MP94] have defined a subgroup $\mathbf{G}_{x, r}$ of $\mathbf{G}$. We abbreviate $\mathbf{G}_{x, 0}$ to $\mathbf{G}_{x}$, a parahoric subgroup of $\mathbf{G}$. Viewing $\mathbf{G}_{x, r}$ as a pro-algebraic group over $k$, its Lie algebra can be identified with $\mathfrak{g}_{x, r}$. The subgroups $\mathbf{G}_{x, r}$ are normal in $\mathbf{G}_{x}$. For $s \geq r$ we have $\mathbf{G}_{x, s} \subset \mathbf{G}_{x, r}$. We write $\mathbf{G}_{x, r+}$ for $\bigcup_{s>r} \mathbf{G}_{x, s}$, a subgroup of $\mathbf{G}_{x, r}$; for sufficiently small $\delta>0 \mathbf{G}_{x, r+\delta}$ is equal to $\mathbf{G}_{x, r+}$. Put $\mathbf{G}_{x}(r):=\mathbf{G}_{x, r} / \mathbf{G}_{x, r+}$.

Let $V$ be a representation of $G$ and let $s$ be a real number. Then the parahoric subgroup $\mathbf{G}_{x}$ preserves $\mathbf{V}_{x, s}$. Moreover, for every $r \geq 0$ the subgroup $\mathbf{G}_{x, r}$ acts trivially on $\mathbf{V}_{x, s} / \mathbf{V}_{x, s+r}$. Finally, for $r>0$ there is a canonical isomorphism

$$
\xi_{x, r}: \mathbf{G}_{x}(r) \rightarrow \mathfrak{g}_{x}(r)
$$

and this isomorphism has the following property. Let $g \in \mathbf{G}_{x, r}$ and let $v \in \mathbf{V}_{x, s}$. Then $g v-v$ lies in $\mathbf{V}_{x, r+s}$, and its image $w$ in $\mathbf{V}_{x}(r+s)$ depends only on the 
image $\bar{g}$ of $g$ in $\mathbf{G}_{x}(r)$ and the image $v_{s}$ of $v$ in $\mathbf{V}_{x}(s)$. The property of $\xi_{x, r}$ we are referring to is that

$$
w=\xi_{x, r}(\bar{g}) \cdot v_{s}
$$

(The right-hand side of this equation uses the map (3.1.1) induced by the action of $\mathfrak{g}$ on V.)

3.3. More filtrations and vector bundles. We will now see that the affine setup of 3.1, 3.2 produces Hessenberg varieties and vector bundles of the type studied in 2.7 by

Suppose we have $x, y \in \mathfrak{a}$ and $r, t \in \mathbf{R}$. We define a subspace $\mathbf{F}_{y}^{t} \mathbf{V}_{x}(r)$ of $\mathbf{V}_{x}(r)$

$$
\mathbf{F}_{y}^{t} \mathbf{V}_{x}(r):=\operatorname{im}\left[\mathbf{V}_{y, t} \cap \mathbf{V}_{x, r} \rightarrow \mathbf{V}_{x, r} / \mathbf{V}_{x, r+}=\mathbf{V}_{x}(r)\right]
$$

Applying this construction to $V=\mathfrak{g}$ and $t=0$, we get

$$
\mathbf{F}_{y}^{0} \mathfrak{g}_{x}(r):=\operatorname{im}\left[\mathfrak{g}_{y, 0} \cap \mathfrak{g}_{x, r} \rightarrow \mathfrak{g}_{x, r} / \mathfrak{g}_{x, r+}=\mathfrak{g}_{x}(r)\right] .
$$

Analogously, for $r \geq 0$ we have a subgroup

$$
\mathbf{F}_{y}^{0} \mathbf{G}_{x}(r):=\operatorname{im}\left[\mathbf{G}_{y} \cap \mathbf{G}_{x, r} \rightarrow \mathbf{G}_{x, r} / \mathbf{G}_{x, r+}=\mathbf{G}_{x}(r)\right],
$$

and for $r>0$ the subgroup $\mathbf{F}_{y}^{0} \mathbf{G}_{x}(r)$ goes over to $\mathbf{F}_{y}^{0} \mathfrak{g}_{x}(r)$ under the isomorphism $\xi_{x, r}: \mathbf{G}_{x}(r) \rightarrow \mathfrak{g}_{x}(r)$.

Put $H_{0}:=\mathbf{G}_{x}(0)$, a connected reductive group over $k$, and put $P=\mathbf{F}_{y}^{0} \mathbf{G}_{x}(0)$, a parabolic subgroup of $H_{0}$. Each space $\mathbf{V}_{x}(r)$ is a representation of $H_{0}$, and $P$ preserves the subspace $\mathbf{F}_{y}^{t} \mathbf{V}_{x}(r)$. Therefore, as in Section 2, we may define an $H_{0^{-}}$ equivariant vector bundle $\mathbf{E}_{y}^{t} \mathbf{V}_{x}(r)$ over $\mathcal{P}:=H_{0} / P$ whose fiber over $h P$ is equal to $h \mathbf{F}_{y}^{t} \mathbf{V}_{x}(r)$.

To see that these constructions are instances of ones made in 2.7, let us examine $\mathbf{F}_{y}^{t} \mathbf{V}_{x}(r)$ more closely. This subspace of $\mathbf{V}_{x}(r)$ is the direct sum of the spaces $V_{\lambda} \epsilon^{m}$ for $(\lambda, m)$ such that $\lambda(x)+m=r$ and $\lambda(y)+m \geq t$. These two conditions can be rewritten as $\lambda(x)+m=r$ and $\lambda(y-x) \geq t-r$, which shows that under the isomorphism $i_{x, r}$ from $\mathbf{V}_{x}(r)$ to $V(x, \mathbf{r})$, the subspace $\mathbf{F}_{y}^{t} \mathbf{V}_{x}(r)$ goes over to $\mathbf{F}_{y-x}^{t-r} V(x, \mathbf{r})$. In particular, under the isomorphism $\mathfrak{g}_{x}(r)=\mathfrak{g}(x, \mathbf{r})$, the subspace $\mathbf{F}_{y}^{0} \mathfrak{g}_{x}(r)$ goes over to $F_{y-x}^{-r} \mathfrak{g}(x, \mathbf{r})$.

Note that $H_{0}$ can be identified with the group $H$ defined in 2.7 (for the given $x \in \mathfrak{a})$, and that this identification is compatible with the identification $\mathfrak{g}_{x}(0)=$ $\mathfrak{g}(x, \mathbf{0})$ of their Lie algebras. Under this identification, the parabolic subgroup $P \subset H_{0}$ goes over to $P_{y-x} \subset H$, and the representation $\mathbf{V}_{x}(r)$ of $H_{0}$ goes over to the representation $V(x, \mathbf{r})$ of $H$. We may also identify $\mathcal{P}=H_{0} / P$ with $\mathcal{P}_{y-x}$, and then the vector bundles $\mathbf{E}_{y}^{t} \mathbf{V}_{x}(r), \mathbf{E}_{y}^{0} \mathfrak{g}_{x}(r)$ go over to $E_{y-x}^{t-r} V(x, \mathbf{r}), E_{y-x}^{-r} \mathfrak{g}(x, \mathbf{r})$ respectively.

\section{Generalized affine Springer fibers}

4.1. Definition of generalized affine Springer fibers. Fix $y \in \mathfrak{a}$. We write $\mathcal{F}_{y}$ for the (partial) affine flag space $\mathbf{G} / \mathbf{G}_{y}$, an ind-scheme over $k$. For $t \in \mathbf{R}$ and $v \in \mathbf{V}$ we define the generalized affine Springer fiber $\mathcal{F}_{y}(t, v) \subset \mathcal{F}_{y}$ by

$$
\mathcal{F}_{y}(t, v)=\left\{g \in \mathbf{G} / \mathbf{G}_{y}: g^{-1} v \in \mathbf{V}_{y, t}\right\},
$$

a closed subset of $\mathcal{F}_{y}$. 
4.2. $t$ and $v$. We are going to analyze the structure of this generalized affine Springer fiber under the following hypotheses on $t, v$. We suppose that there exist $x \in \mathfrak{a}$ and $s \in \mathbf{R}$ such that

(1) $s \geq t$,

(2) $v \in \mathbf{V}_{x, s}$, and

(3) the image $\bar{v}$ of $v$ under $\mathbf{V}_{x, s} \rightarrow \mathbf{V}_{x}(s) \rightarrow V(x, \mathbf{s}) \hookrightarrow V$ is a $G$-good vector in $V$.

In the third condition the map $\mathbf{V}_{x, s} \rightarrow \mathbf{V}_{x}(s)$ is the canonical projection and the map $\mathbf{V}_{x}(s) \rightarrow V(x, \mathbf{s})$ is the linear isomorphism $i_{x, s}$ defined in 3.1 .

4.3. Intersection with orbits. In order to analyze $\mathcal{F}_{y}(t, v)$ we intersect it with the orbits of $\mathbf{G}_{x}$ in $\mathcal{F}_{y}$. The intersections will turn out to be iterated affine space bundles over Hessenberg varieties (and are therefore empty precisely when the Hessenberg variety is empty).

We first make the observation that it is enough to consider the orbit of the basepoint in $\mathcal{F}_{y}$. Indeed, right multiplication by an element $c$ in the normalizer of $A(F)$ in $G(F)$ induces an isomorphism from the intersection of $\mathcal{F}_{c y}(t, v)$ and the $\mathbf{G}_{x^{-}}$orbit of the base-point in $\mathcal{F}_{c y}$ to the intersection of $\mathcal{F}_{y}(t, v)$ and the $\mathbf{G}_{x}$-orbit of $c$ times the base-point in $\mathcal{F}_{y}$. (Recall that $c$ acts on $\mathfrak{a}$ by an affine linear transformation; $c y$ denotes the result of this action on $y$.)

The intersection of $\mathcal{F}_{y}(t, v)$ and the $\mathbf{G}_{x}$-orbit of the base-point in $\mathcal{F}_{y}$ is equal to

$$
S:=\left\{g \in \mathbf{G}_{x} /\left(\mathbf{G}_{x} \cap \mathbf{G}_{y}\right): g^{-1} v \in \mathbf{V}_{y, t}\right\} .
$$

4.4. Spaces $\tilde{S}_{r}$ and $S_{r}$. We must analyze $S$, and in order to do so we introduce some auxiliary spaces. For $r>0$ put

$$
\tilde{S}_{r}:=\left\{g \in \mathbf{G}_{x} /\left(\mathbf{G}_{x} \cap \mathbf{G}_{y}\right): g^{-1} v \in \mathbf{V}_{y, t}+\mathbf{V}_{x, s+r}\right\}
$$

and for $r \geq 0$ put

$$
\tilde{S}_{r+}:=\left\{g \in \mathbf{G}_{x} /\left(\mathbf{G}_{x} \cap \mathbf{G}_{y}\right): g^{-1} v \in \mathbf{V}_{y, t}+\mathbf{V}_{x,(s+r)+}\right\} .
$$

Note that $\tilde{S}_{r+}=\tilde{S}_{r+\delta}$ for all sufficiently small $\delta>0$.

Since $v \in \mathbf{V}_{x, s}$ we have $g^{-1} v \in \mathbf{V}_{x, s}$ for any $g \in \mathbf{G}_{x}$, and the condition $g^{-1} v \in \mathbf{V}_{y, t}+\mathbf{V}_{x, s+r}$ can be thought of as the condition that the image of $g^{-1} v$ in $\mathbf{V}_{x, s} / \mathbf{V}_{x, s+r}$ lie in the subspace

$$
\operatorname{im}\left[\mathbf{V}_{y, t} \cap \mathbf{V}_{x, s} \rightarrow \mathbf{V}_{x, s} / \mathbf{V}_{x, s+r}\right]
$$

Since $\mathbf{G}_{x, r}$ is normal in $\mathbf{G}_{x}$ and acts trivially on $\mathbf{V}_{x, s} / \mathbf{V}_{x, s+r}$, we see that $\tilde{S}_{r}$ (respectively, $\tilde{S}_{r+}$ ) is invariant under the left action of $\mathbf{G}_{x, r}$ (respectively, $\mathbf{G}_{x, r+}$ ), so that we may define quotient spaces

$$
\begin{aligned}
S_{r} & :=\mathbf{G}_{x, r} \backslash \tilde{S}_{r}, \\
S_{r+} & :=\mathbf{G}_{x, r+} \backslash \tilde{S}_{r+} .
\end{aligned}
$$

Note that $S_{r+}=S_{r+\delta}$ for all sufficiently small $\delta>0$.

Let $0=r_{0}<r_{1}<r_{2}<r_{3}<\ldots$ be the discrete set of values of $r \geq 0$ for which either $\mathbf{G}_{x}(r)$ or $\mathbf{V}_{x}(s+r)$ is non-trivial. We have inclusion maps

$$
\cdots \rightarrow \tilde{S}_{r_{3}} \rightarrow \tilde{S}_{r_{2}} \rightarrow \tilde{S}_{r_{1}}
$$

and these induce maps

$$
\cdots \rightarrow S_{r_{3}} \rightarrow S_{r_{2}} \rightarrow S_{r_{1}}
$$


Note that $S_{r_{j}}=S$ for sufficiently large $j$, and that $S_{r_{i+1}}=S_{r_{i}+}$ for all $i \geq 0$ (in particular, $S_{r_{1}}=S_{0+}$ ). Therefore, in order to show that $S$ is an iterated affine space bundle over a Hessenberg variety it is enough to show that $S_{0+}$ is a Hessenberg variety and that for every $r>0$ the map $S_{r+} \rightarrow S_{r}$ is an affine space bundle. In fact we will prove a more precise statement. For each $r \geq 0$ we are going to construct a vector bundle $E_{r}$ over the Hessenberg variety $S_{0+}$. It will turn out that $E_{0}$ is the tangent bundle to $S_{0+}$ and that $S_{r+} \rightarrow S_{r}$ is a torsor under the vector bundle on $S_{r}$ obtained by pulling back the vector bundle $E_{r}$ by means of $S_{r} \rightarrow S_{0+}$. Thus the dimension of $S$ is given by $\sum_{r \geq 0} \operatorname{dim} E_{r}$, the sum having only finitely many non-zero terms. However, it is conceptually clearer to calculate the dimension slightly differently, as we will do in 4.8 below.

4.5. Analysis of $S_{0+}$. We now check that $S_{0+}$ is a Hessenberg variety. We use the notation of 3.3. It is immediate from the definitions that

$$
S_{0+}=\left\{g \in H_{0} / P: g^{-1} v_{s} \in \mathbf{F}_{y}^{t} \mathbf{V}_{x}(s)\right\}
$$

where $v_{s}$ denotes the image of $v$ under the canonical projection $\mathbf{V}_{x, s} \rightarrow \mathbf{V}_{x}(s)$. It follows from the discussion in 3.3 that $S_{0+}$ equals $\mathcal{P}_{y-x}(t-s, \bar{v})$, where $\bar{v}$ is the image of $v_{s}$ under the isomorphism $i_{x, s}: \mathbf{V}_{x}(s) \rightarrow V(x, \mathbf{s})$. Since $t-s \leq 0$ and $\bar{v}$ is $G$-good by hypothesis, it follows that $\mathcal{P}_{y-x}(t-s, \bar{v})$ is a Hessenberg variety.

4.6. Some auxiliary bundles. Fix a real number $r>0$. Before analyzing the fibers of $S_{r+} \rightarrow S_{r}$, we introduce some auxiliary bundles. Consider the map

$$
p: \mathbf{G}_{x, r+} \backslash \mathbf{G}_{x} \rightarrow \mathbf{G}_{x, r} \backslash \mathbf{G}_{x} .
$$

Since $\mathbf{G}_{x}(r)=\mathfrak{g}_{x}(r)$, it is clear that $p$ is a principal $\mathfrak{g}_{x}(r)$-bundle, or in other words, a torsor under the constant vector bundle $\tilde{\mathfrak{g}}_{x}(r)$ over $\mathbf{G}_{x, r} \backslash \mathbf{G}_{x}$ with fiber $\mathfrak{g}_{x}(r)$.

Next consider the map

$$
q: \mathbf{G}_{x, r+} \backslash \mathbf{G}_{x} /\left(\mathbf{G}_{x} \cap \mathbf{G}_{y}\right) \rightarrow \mathbf{G}_{x, r} \backslash \mathbf{G}_{x} /\left(\mathbf{G}_{x} \cap \mathbf{G}_{y}\right) .
$$

The target of $q$ maps to

$$
\mathbf{G}_{x, 0+} \backslash \mathbf{G}_{x} /\left(\mathbf{G}_{x} \cap \mathbf{G}_{y}\right)=H_{0} / P=\mathcal{P} .
$$

On $\mathcal{P}$ we have the vector bundle $\mathbf{E}_{y}^{0} \mathfrak{g}_{x}(r)$, and it is easy to see that $q$ is a torsor under the quotient of $\tilde{\mathfrak{g}}_{x}(r)$ by the pullback of $\mathbf{E}_{y}^{0} \mathfrak{g}_{x}(r)$.

4.7. Analysis of $S_{r+} \rightarrow S_{r}$. We continue with $r$ and $q$ as in 4.6. Of course $S_{r+}$ is a closed subspace of the source of $q$ and $S_{r}$ is a closed subspace of the target of $q$, and it is the restriction of $q$ to $S_{r+}$ that gives the map $S_{r+} \rightarrow S_{r}$ that we need to understand.

In order to see that $S_{r+} \rightarrow S_{r}$ is a bundle, we begin by making the following simple calculation. Suppose that $g \in \mathbf{G}_{x}$ represents an element of $S_{r}$, so that $g^{-1} v \in \mathbf{V}_{y, t}+\mathbf{V}_{x, s+r}$. Then $g$ represents an element of $S_{r+}$ if and only if the image of $v$ is 0 in

$$
\frac{g \mathbf{V}_{y, t}+\mathbf{V}_{x, s+r}}{g \mathbf{V}_{y, t}+\mathbf{V}_{x,(s+r)+}}=\frac{\mathbf{V}_{x}(s+r)}{g \mathbf{F}_{y}^{t} \mathbf{V}_{x}(s+r)}
$$

Suppose that we replace $g$ by $g_{r} g$ with $g_{r} \in \mathbf{G}_{x, r}$. (In other words, we are considering points in the fiber of $q$ through the point represented by $g$.) Note that

$$
g_{r} g \mathbf{F}_{y}^{t} \mathbf{V}_{x}(s+r)=g \mathbf{F}_{y}^{t} \mathbf{V}_{x}(s+r) \text {. }
$$


Since $v \in \mathbf{V}_{x, s}$ we have $g_{r}^{-1} v \equiv v-\xi_{x, r}\left(g_{r}\right) \cdot v$ modulo $\mathbf{V}_{x,(r+s)+}$. Therefore, the element of $\mathbf{V}_{x}(s+r) / g \mathbf{F}_{y}^{t} \mathbf{V}_{x}(s+r)$ obtained from $g_{r} g$ differs from the one obtained from $g$ by the element $\xi_{x, r}\left(g_{r}\right) \cdot v_{s} \in \mathbf{V}_{x}(s+r)$, where $v_{s}$ denotes the image of $v$ in $\mathbf{V}_{x}(s)$. Therefore, $g \in S_{r}$ lies in the image of $S_{r+}$ if and only if the image of $v$ in

$$
\frac{\mathbf{V}_{x}(s+r)}{g \mathbf{F}_{y}^{t} \mathbf{V}_{x}(s+r)}
$$

lies in the image of

$$
\cdot v_{s}: \mathfrak{g}_{x}(r) \rightarrow \frac{\mathbf{V}_{x}(s+r)}{g \mathbf{F}_{y}^{t} \mathbf{V}_{x}(s+r)}
$$

Using the linear isomorphisms $\mathfrak{g}_{x}(r) \simeq \mathfrak{g}(x, \mathbf{r})$ and $\mathbf{V}_{x}(s+r) \simeq V(x, \mathbf{s}+\mathbf{r})$ discussed at the end of 3.1, we can rewrite this last map as

$$
\cdot \bar{v}: \mathfrak{g}(x, \mathbf{r}) \rightarrow V(x, \mathbf{s}+\mathbf{r}) / \bar{g} F_{y-x}^{t-s-r} V(x, \mathbf{s}+\mathbf{r}),
$$

where $\bar{g}$ denotes the image of $g$ in $\mathbf{G}_{x}(0)=H_{0}$. (Recall from 3.3 that $H_{0}$ can be identified with the group $H$ defined in 2.7 and that $V(x, \mathbf{s}+\mathbf{r})$ is a representation of $H$.) Our hypotheses guarantee that $t-s-r<0$ and that $\bar{v}$ is a $G$-good vector in $V$. It then follows from 2.7 that the map $\cdot \bar{v}$ above is surjective, and hence that $g \in S_{r}$ does lie in the image of $S_{r+}$.

Therefore, $S_{r+} \rightarrow S_{r}$ is surjective, and the analysis above (together with 4.6) shows that the fiber of $S_{r+} \rightarrow S_{r}$ through the point in $S_{r+}$ represented by $g \in \mathbf{G}_{x}$ is equal to the kernel of the map $\cdot \bar{v}$ above modulo $g \mathbf{F}_{y}^{0} \mathfrak{g}_{x}(r)=\bar{g} F_{y-x}^{-r} \mathfrak{g}(x, \mathbf{r})$. This shows that $S_{r+} \rightarrow S_{r}$ is a torsor under the vector bundle on $S_{r}$ obtained by pulling back the vector bundle

$$
\begin{aligned}
E_{r}: & =\operatorname{ker}\left[\cdot v_{s}: \tilde{\mathfrak{g}}_{x}(r) / \mathbf{E}_{y}^{0} \mathfrak{g}_{x}(r) \rightarrow \tilde{\mathbf{V}}_{x}(s+r) / \mathbf{E}_{y}^{t} \mathbf{V}_{x}(s+r)\right] \\
& =\operatorname{ker}\left[\bar{v}: \tilde{\mathfrak{g}}(x, \mathbf{r}) / E_{y-x}^{-r} \mathfrak{g}(x, \mathbf{r}) \rightarrow \tilde{V}(x, \mathbf{s}+\mathbf{r}) / E_{y-x}^{t-s-r} V(x, \mathbf{s}+\mathbf{r})\right],
\end{aligned}
$$

the vector bundle denoted by $K(x, y-x, r, s, t-s,-r, \bar{v})$ in 2.7 Note that the vector bundle $E_{r}$ is also defined for $r=0$, in which case it coincides with the tangent bundle (see 2.5) of the Hessenberg variety $S_{0+}=\mathcal{P}_{y-x}(t-s, \bar{v})$.

4.8. Dimension of $S$. Now we are going to calculate the dimension of $S$. The group $\mathbf{G}_{x}$ acts on the vector space $\mathbf{V}_{x, s}$. Over $\mathbf{G}_{x} /\left(\mathbf{G}_{x} \cap \mathbf{G}_{y}\right)$ we have the vector bundle whose fiber at $g \in \mathbf{G}_{x} /\left(\mathbf{G}_{x} \cap \mathbf{G}_{y}\right)$ is the vector space $\mathbf{V}_{x, s} /\left(\mathbf{V}_{x, s} \cap g \mathbf{V}_{y, t}\right)$, and the vector $v \in \mathbf{V}_{x, s}$ determines a section of this vector bundle, a section whose zero-set coincides with $S$, as follows immediately from the definitions.

In fact this section is transverse to the zero-section. In other words, the map

$$
\cdot v: \mathfrak{g}_{x} \rightarrow \mathbf{V}_{x, s} /\left(\mathbf{V}_{x, s} \cap g \mathbf{V}_{y, t}\right)
$$

is surjective for every $g \in \mathbf{G}_{x}$ that represents a point in $S$, as follows from the surjectivity of the maps (4.7.1). Transversality implies that

$$
\operatorname{dim}(S)=\operatorname{dim}\left(\mathfrak{g}_{x} /\left(\mathfrak{g}_{x} \cap \mathfrak{g}_{y}\right)\right)-\operatorname{dim}\left(\mathbf{V}_{x, s} /\left(\mathbf{V}_{x, s} \cap \mathbf{V}_{y, t}\right)\right) .
$$

It is clear that $\operatorname{dim}\left(\mathfrak{g}_{x} /\left(\mathfrak{g}_{x} \cap \mathfrak{g}_{y}\right)\right)$ is the number of affine roots that are non-negative on $x$ and strictly negative on $y$. In the important special case when $\mathbf{V}=\mathfrak{g}$ and $t=0$, there is a similar expression for the last dimension appearing in equation (4.8.1), and we conclude that in this special case the dimension of $S$ is the cardinality of

$$
\{\text { affine roots } \tilde{\alpha}: 0 \leq \tilde{\alpha}(x)<s \text { and } \tilde{\alpha}(y)<0\} .
$$




\section{Affine Springer fibers in the Equivalued CASe}

5.1. Additional hypothesis on $k$. Throughout this section we assume that the order of the Weyl group $W:=N_{G}(A) / A$ of $G$ is invertible in $k$.

5.2. G-conjugacy classes of maximal $F$-tori in $G_{F}$. We write $G_{F}$ for the $F$ group obtained from $G$ by extension of scalars from $k$ to $F$, and as before we write $\mathbf{G}$ for $G(F)$. We need to review the well-known classification of $\mathbf{G}$-conjugacy classes of maximal $F$-tori in $G$.

Let $T$ be a maximal $F$-torus in $G_{F}$. Fix an algebraic closure $\bar{F}$ of $F$ and let $F_{s}$ denote the separable closure of $F$ in $\bar{F}$. Choose $h \in G\left(F_{s}\right)$ such that $T=h A h^{-1}$. For $\sigma \in \Gamma:=\operatorname{Gal}\left(F_{s} / F\right)$ put $x_{\sigma}=h^{-1} \sigma(h)$. Then $\sigma \mapsto x_{\sigma}$ is a 1-cocycle of $\Gamma$ in $G\left(F_{s}\right)$, whose class in $H^{1}\left(F, N_{G}(A)\right)$ we denote by $x(T)$. This construction yields a bijection from the set of G-conjugacy classes of maximal $F$-tori in $G_{F}$ to the set

$$
\operatorname{ker}\left[H^{1}\left(F, N_{G}(A)\right) \rightarrow H^{1}(F, G)\right] .
$$

The obvious surjection $N_{G}(A) \rightarrow W$ induces an injection

$$
H^{1}\left(F, N_{G}(A)\right) \rightarrow H^{1}(F, W)
$$

since for our particular field $F$ it is known Ser68 that $H^{1}\left(F, T^{\prime}\right)$ vanishes for every $F$-torus $T^{\prime}$. Denoting by $y(T)$ the image of $x(T)$ in $H^{1}(F, W)$, we obtain an injection $T \mapsto y(T)$ from the set of $\mathbf{G}$-conjugacy classes of maximal $F$-tori in $G_{F}$ to $H^{1}(F, W)$.

In fact all elements of $H^{1}(F, W)$ arise in this way, and we will now review a proof of this fact, since for our purposes we need to produce an explicit maximal $F$-torus $T$ for which $y(T)$ is a given element of $H^{1}(F, W)$.

5.3. Construction of $T$. We start with a 1-cocycle of $\Gamma$ in $W$ and we will construct $T$ such that $y(T)$ is the class of this 1-cocycle. Since the Galois action on $W$ is trivial, the 1-cocycle is simply a homomorphism $\Gamma \rightarrow W$.

For any integer $l$ not divisible by the characteristic of $k$ we let $F_{l}$ denote the subfield of $F_{s}$ obtained by adjoining an $l$-th root $\epsilon^{1 / l}$ of $\epsilon$ to $F$, and we fix a primitive $l$-th root $\zeta_{l}$ of 1 in $k$. We do this in such a way that $\zeta_{l m}^{m}=\zeta_{l}$ for all positive integers $l, m$ not divisible by the characteristic. Recall that $\operatorname{Gal}\left(F_{l} / F\right)$ is cyclic of order $l$, generated by the automorphism $\tau_{l}$ sending $\epsilon^{1 / l}$ to $\zeta_{l} \epsilon^{1 / l}$.

Since $|W|$ is not divisible by the characteristic, the given homomorphism $\Gamma \rightarrow W$ comes from a homomorphism $\varphi: \operatorname{Gal}\left(F_{|W|} / F\right) \rightarrow W$, and we denote by $w$ the element $\varphi\left(\tau_{|W|}\right) \in W$. Choose an element $\dot{w} \in N_{G}(A)(k)$ of finite order such that $\dot{w} \mapsto w$. We denote by $l$ the order of $\dot{w}$. Note that $l$ is not divisible by the characteristic. Thus there is a homomorphism $\operatorname{Gal}\left(F_{l} / F\right) \rightarrow N_{G}(A)(k)$ sending $\tau_{l}$ to $\dot{w}$, and this homomorphism lifts the given homomorphism $\operatorname{Gal}\left(F_{|W|} / F\right) \rightarrow W$.

The element $\dot{w} \in G(k)$ is semisimple, so there exists a maximal torus $A^{\prime}$ in $G$ such that $\dot{w} \in A^{\prime}(k)$. We have lifted our 1-cocycle in $W$ to a 1-cocycle in $N_{G}(A)$ that takes values in $A^{\prime}$. In order to produce $T$ we need to write this 1-cocycle as the coboundary of an element in $G\left(F_{s}\right)$.

But our 1-cocycle happens to take values in the split torus $A^{\prime}$, so by Hilbert's Theorem 90, it must be possible to construct an element $b \in A^{\prime}\left(F_{l}\right)$ such that $b^{-1} \tau_{l}(b)=\dot{w}$. To obtain an explicit element $b$, we note that since $\dot{w}$ is an $l$ torsion element in $A^{\prime}(k)$, there exists $\mu \in X_{*}\left(A^{\prime}\right)$ such that $\mu\left(\zeta_{l}^{-1}\right)=\dot{w}$. Now put $b=\mu\left(\epsilon^{1 / l}\right)^{-1} \in A^{\prime}\left(F_{l}\right)$; an easy calculation shows that $b^{-1} \tau_{l}(b)=\dot{w}$, as desired. 
Define a maximal torus $T$ by $T:=b A b^{-1}$. Since $b^{-1} \tau_{l}(b)=\dot{w}$, it follows that $T$ is defined over $F$ and that $y(T)$ is the class in $H^{1}(F, W)$ we started with. This completes the explicit construction of $T$.

5.4. Comparison of two filtrations on $\mathbf{t}$. The appropriate context here is that of apartments for tamely ramified tori in reductive groups over non-archimedean local fields (see Ad198, Yu01 for example).

We continue with the maximal torus we just constructed. We use the maximal torus $A^{\prime}$ and the element $x:=\mu / l \in X_{*}\left(A^{\prime}\right) \otimes \mathbf{z} \mathbf{R}$ to obtain an $\mathbf{R}$-filtration $\mathfrak{g}_{x, s}$ $(s \in \mathbf{R})$. It is one of the Moy-Prasad filtrations considered before, but for $A^{\prime}$ rather than $A$.

Let $\mathbf{t}$ denote the Lie algebra of $T$, a Cartan subalgebra of $\mathfrak{g}$. We want to understand the filtration on $\mathbf{t}$ induced by the filtration $\mathfrak{g}_{x, s}$ on $\mathfrak{g}$. To do so we first extend the filtration $\mathfrak{g}_{x, s}$ on $\mathfrak{g}$ to a filtration on $\mathfrak{g}\left(F_{l}\right):=\mathfrak{g} \otimes_{k} F_{l}$ by putting

$$
\mathfrak{g}\left(F_{l}\right)_{x}(s):=\prod_{(\lambda, m)} \mathfrak{g}_{\lambda} \cdot\left(\epsilon^{1 / l}\right)^{m}
$$

where the index set for the product is the set of $(\lambda, m) \in X^{*}\left(A^{\prime}\right) \oplus \mathbf{Z}$ such that $\lambda(x)+(m / l)=s$, or equivalently $\langle\lambda, \mu\rangle+m=s l$, and then putting

$$
\mathfrak{g}\left(F_{l}\right)_{x, s}:=\prod_{s^{\prime} \geq s} \mathfrak{g}\left(F_{l}\right)_{x}\left(s^{\prime}\right) .
$$

Similarly, we extend the filtration $\mathfrak{g}_{0, s}$ on $\mathfrak{g}$ to a filtration on $\mathfrak{g}\left(F_{l}\right)$ by putting

$$
\mathfrak{g}\left(F_{l}\right)_{0}(s):=\mathfrak{g} \cdot\left(\epsilon^{1 / l}\right)^{m}
$$

for $s$ of the form $m / l$ for $m \in \mathbf{Z}$ and setting it equal to 0 otherwise, and then putting

$$
\mathfrak{g}\left(F_{l}\right)_{0, s}:=\prod_{s^{\prime} \geq s} \mathfrak{g}\left(F_{l}\right)_{0}\left(s^{\prime}\right) .
$$

The group $k^{\times}$acts on $F_{l}$ by automorphisms over $k$. We denote by $\tau_{z} \in \operatorname{Aut}\left(F_{l} / k\right)$ the automorphism obtained from $z \in k^{\times}$; it sends $\epsilon^{1 / l}$ to $z \epsilon^{1 / l}$. This action induces an action $z \mapsto \operatorname{id}_{\mathfrak{g}} \otimes \tau_{z}$ of $k^{\times}$on $\mathfrak{g}\left(F_{l}\right)$, and we also denote this action by $\tau_{z}$. Using $\mu$, we get a new action $\tau_{z}^{\prime}$ of $k^{\times}$on $\mathfrak{g}\left(F_{l}\right)$, for which $z \in k^{\times}$acts by $\operatorname{Ad}(\mu(z)) \circ \tau_{z}$. For the action $\tau_{z}^{\prime}$ an element $z \in k^{\times}$acts on $\mathfrak{g}_{\lambda} \cdot\left(\epsilon^{1 / l}\right)^{m}$ by $z^{\langle\lambda, \mu\rangle+m}$, which shows that $\mathfrak{g}\left(F_{l}\right)_{x, s}$ is the product of the weight spaces for the $\tau_{z}^{\prime}$ action for weights $j \in \mathbf{Z}$ such that $j \geq s l$. Similarly, $\mathfrak{g}\left(F_{l}\right)_{0, s}$ is the product of the weight spaces for the $\tau_{z}$ action for weights $j \in \mathbf{Z}$ such that $j \geq s l$.

An easy calculation shows that

$$
\tau_{z}^{\prime}=\operatorname{Ad}(b) \circ \tau_{z} \circ \operatorname{Ad}(b)^{-1} .
$$

Therefore $\operatorname{Ad}(b)^{-1} \mathfrak{g}\left(F_{l}\right)_{x, s}=\mathfrak{g}\left(F_{l}\right)_{0, s}$. Moreover, $b^{-1} T b=A$. This shows that when we use $\operatorname{Ad}(b)^{-1}$ to identify $\operatorname{Lie}\left(T_{F_{l}}\right)$ with $\operatorname{Lie}\left(A_{F_{l}}\right)$, the subspaces $\mathfrak{g}\left(F_{l}\right)_{x, s} \cap \operatorname{Lie}\left(T_{F_{l}}\right)$ and $\mathfrak{g}\left(F_{l}\right)_{x}(s) \cap \operatorname{Lie}\left(T_{F_{l}}\right)$ go over to $\mathfrak{g}\left(F_{l}\right)_{0, s} \cap \operatorname{Lie}\left(A_{F_{l}}\right)$ and $\mathfrak{g}\left(F_{l}\right)_{0}(s) \cap \operatorname{Lie}\left(A_{F_{l}}\right)$ respectively. Note that $\mathfrak{g}\left(F_{l}\right)_{0}(s) \cap \operatorname{Lie}\left(A_{F_{l}}\right)$ is $\operatorname{Lie}(A) \cdot\left(\epsilon^{1 / l}\right)^{m}$ if $s=m / l$ for some $m \in \mathbf{Z}$ and is 0 otherwise. 
5.5. Equivalued elements. We continue with $T$ as above. Let val $: F_{l}{ }^{\times} \rightarrow \mathbf{Q}$ be the valuation on $F_{l}$, normalized so that $\operatorname{val}\left(\epsilon^{1 / l}\right)=1 / l$. Let $u \in \mathbf{t}$ and suppose that $u$ is regular, so that $\alpha(u) \in F_{l}^{\times}$for every root $\alpha$ of $T$ (over $\bar{F}$ ). Suppose further that there exists $s \in \mathbf{Q}$ such that $\operatorname{val} \alpha(u)=s$ for every root $\alpha$ of $T$ (over $\bar{F}$ ) and $\operatorname{val} \lambda(u) \geq s$ for every $\lambda \in X^{*}(T)$, in which case we say that $u$ is equivalued with valuation $s$. (Here we are using the convention that val $0=+\infty$.)

This hypothesis on $u$ can also be rephrased as saying that $u^{\prime}=\operatorname{Ad}(b)^{-1} u \in$ $\prod_{m \geq l s} \operatorname{Lie}(A) \cdot\left(\epsilon^{1 / l}\right)^{m}$ and that the projection of $u^{\prime}$ on $\operatorname{Lie}(A) \cdot\left(\epsilon^{1 / l}\right)^{l s}$ is of the form $u^{\prime \prime} \cdot\left(\epsilon^{1 / l}\right)^{l s}$ with $u^{\prime \prime}$ regular in $\operatorname{Lie}(A)$. It follows from our comparison of filtrations in 5.4 that $u \in \mathfrak{g}_{x, s}$ and that the image of $u$ under

$$
\mathfrak{g}_{x, s} \rightarrow \mathfrak{g}_{x}(s)=\mathfrak{g}(x, \mathbf{s}) \hookrightarrow \mathfrak{g},
$$

namely $u^{\prime \prime}$, is regular semisimple in $\mathfrak{g}$, hence is $G$-good in $\mathfrak{g}$ (see 2.3 noting that if $W$ is non-trivial, its order is divisible by 2, so that characteristic 2 has been excluded).

A minor nuisance is that $x$ lies in $X_{*}\left(A^{\prime}\right) \otimes_{\mathbf{z}} \mathbf{R}$ rather than $\mathfrak{a}$. We can correct this by replacing $T, u$ by their conjugates under an element of $G(k)$ that takes $A^{\prime}$ to $A$. This replaces $x$ by an element of $\mathfrak{a}$, which we will still call $x$. For this new $x$ and for any $t \in \mathbf{R}$ with $t \leq s$ we have shown that the three conditions of 4.2 are satisfied. We conclude that for any $y \in \mathfrak{a}$ and $t \leq s$ the intersection of the affine Springer fiber $\mathcal{F}_{y}(t, u)$ with any $\mathbf{G}_{x}$-orbit on $\mathcal{P}_{y}$ is an iterated affine space bundle over a Hessenberg variety for $\mathbf{G}_{x}(0)$. (The Hessenberg variety depends on the orbit.) This proves Theorem 1.1 .

5.6. Weakly Coxeter tori. For convenience we now assume that the maximal $F$-split torus $A_{T}$ in $T$ is contained in $A$, so that the centralizer $M$ of $A_{T}$ is a Levi subgroup of $G$ (over $k$ ) containing $A$. We further assume that $T$ is weakly Coxeter, so that $w$ is a Coxeter element in $W_{M}$, the absolute Weyl group of $A$ in $M$. Our lifting $\dot{w}$ of $\$ 5.3$ is a regular semisimple element in $M$ by a result of Kostant [Kos59]. We are free to modify $\dot{w}$ by any element in the center of $M$; doing so we may choose $\dot{w}$ so that it is regular in $G$, not just in $M$. Looking back at how $x$ was defined in $\$ 5.4$, we see that $x$ lies in the interior of an alcove. Thus $\mathbf{G}_{x}$ is an Iwahori subgroup, and the Hessenberg varieties we get are subvarieties of the flag manifold of the torus $\mathbf{G}_{x}(0)$ and hence have either 1 or 0 elements. Thus, for weakly Coxeter $T$ our Hessenberg paving is a paving by affine spaces. This proves Theorem 1.2 ,

\section{Regular SEMisimple IMPLies GOOD WHEN $p \neq 2$}

In this section we fix a prime number $p$ and assume that our algebraically closed ground field $k$ has characteristic $p$.

6.1. Review of regularity in $\mathfrak{g}$. Recall from [Bor69, Prop. 11.8] that $u \in \mathfrak{g}$ is semisimple if and only if it is contained in the Lie algebra of some maximal torus $T$ of $G$, in which case it is clear that the centralizer $\mathfrak{g}_{u}$ of $u$ in $\mathfrak{g}$ contains the Lie algebra of that maximal torus. A semisimple element $u \in \mathfrak{g}$ is said to be regular if its centralizer is as small as possible, or, in other words, is equal to the Lie algebra of some maximal torus. We denote by $\mathfrak{g}_{\mathrm{rs}}$ the set of regular semisimple elements in $\mathfrak{g}$. 
6.2. Non-emptiness of $\mathfrak{g}_{\mathrm{rs}}$. Consider elements $u$ in the Lie algebra $\mathfrak{t}$ of some fixed maximal torus $T$ in $G$. Then $u$ is regular if and only $\alpha(u) \neq 0$ for every root $\alpha$ of $T$ in $G$. By conjugacy of maximal tori, $\mathfrak{g}_{\mathrm{rs}}$ is non-empty if and only if the set of regular semisimple elements in $\mathfrak{t}$ is non-empty, and this happens if and only if no root of $T$ vanishes identically on $\mathfrak{t}$. It even suffices to look only at simple roots, since any root is in the Weyl group orbit of a simple one. (A root vanishes identically on $\mathfrak{t}$ if and only if it is divisible by $p$ in $X^{*}(T)$.)

If $G$ is adjoint, no simple root is divisible by $p$, and therefore $\mathfrak{g}_{\mathrm{rs}}$ is non-empty. On the other hand, $\mathfrak{g}_{\mathrm{rs}}$ is in fact empty for $G=S p_{2 n}$ and $p=2$. Since $\left\langle\alpha^{\vee}, \alpha\right\rangle=2$, a root is never divisible by a prime other than 2 ; therefore $\mathfrak{g}_{\mathrm{rs}}$ is non-empty if $p \neq 2$.

6.3. Chevalley's restriction theorem. Chevalley's theorem states that so long as $\mathfrak{g}_{\mathrm{rs}}$ is non-empty, the restriction map from $G$-invariant polynomials on $\mathfrak{g}$ to Weyl group invariant polynomials on $\mathfrak{t}$ is an isomorphism. We refer to Springer and Steinberg [SS70, Section 3.17] for a proof that works in finite characteristic. (Springer and Steinberg assume that $G$ is adjoint, but it is routine to adapt their proof to the more general case in which it is assumed only that $\mathfrak{g}_{\mathrm{rs}}$ is non-empty.)

6.4. Parallel results for $\mathfrak{g}^{*}$. Consider the dual $\mathfrak{g}^{*}$ of $\mathfrak{g}$, a representation of $G$. Since $\mathfrak{t}$ is canonically a direct summand of $\mathfrak{g}$ (with complement the direct sum of the root subspaces for $T$ ), we may identify the dual $\mathfrak{t}^{*}$ of $\mathfrak{t}$ with a direct summand of $\mathfrak{g}^{*}$. For $\lambda \in \mathfrak{t}^{*}$ it is easy to see that the centralizer of $\lambda$ in $\mathfrak{g}$ (for the coadjoint action of $G$ on $\left.\mathfrak{g}^{*}\right)$ is the direct sum of $\mathfrak{t}$ and the root subspaces $\mathfrak{g}_{\alpha}$ for roots $\alpha$ of $T$ such that $\lambda\left(H_{\alpha}\right)=0$. Here $H_{\alpha}:=\alpha^{\vee}(1)$, in other words, the image of the coroot $\alpha^{\vee}$ in $\mathfrak{t}$. An element $\lambda \in \mathfrak{t}^{*}$ is said to be regular if its centralizer is as small as possible, or, in other words, is equal to $\mathfrak{t}$. We denote by $\mathfrak{g}_{\mathrm{rs}}^{*}$ the set of elements in $\mathfrak{g}^{*}$ whose coadjoint orbit contains a regular element of $\mathfrak{t}^{*}$.

Considerations parallel to those above show that $\mathfrak{g}_{\mathrm{rs}}^{*}$ is non-empty if $p \neq 2$ or if $G$ is semisimple simply connected. On the other hand, $\mathfrak{g}_{\mathrm{rs}}^{*}$ is empty for $S O(2 n+1)$ and $p=2$.

Finally, it is routine to transpose the proof of Springer and Steinberg from $\mathfrak{g}$ to $\mathfrak{g}^{*}$, where it yields the statement that if $\mathfrak{g}_{\mathrm{rs}}^{*}$ is non-empty, then the restriction map from $G$-invariant polynomials on $\mathfrak{g}^{*}$ to Weyl group invariant polynomials on $\mathfrak{t}^{*}$ is an isomorphism.

6.5. Goodness of elements in $\mathfrak{g}_{\mathrm{rs}}$ and $\mathfrak{g}_{\mathrm{rs}}^{*}$. Assume that both $\mathfrak{g}_{\mathrm{rs}}$ and $\mathfrak{g}_{\mathrm{rs}}^{*}$ are non-empty. Then Chevalley's restriction theorem holds for both $\mathfrak{g}$ and $\mathfrak{g}^{*}$, and it is therefore clear that there are no non-zero unstable vectors (in the sense of 2.2) in $\mathfrak{t}$ and $\mathfrak{t}^{*}$. It follows easily that elements in $\mathfrak{g}_{\mathrm{rs}}$ and $\mathfrak{g}_{\mathrm{rs}}^{*}$ are good vectors (in the sense of 2.3 .

\section{Conclusions}

In this section we summarize the proof of Theorem 1.1 and outline the standard proof of Corollary 1.3 .

7.1. Summary of Theorem 1.1. The fields $k, F=k((\epsilon))$, the group $G$, and the maximal torus $A$ were fixed at the beginning of the paper. As in $\$ 5.1$ assume the order $|W|$ of the Weyl group for $A$ in $G$ is invertible in $k$.

Let $y \in \mathfrak{a}$. For the representation $V$ (cf. \$2.1) of $G$, take the adjoint representation $V=\mathfrak{g}$. Let $T$ be a maximal torus in $G$ and let $u \in \mathbf{t}$ be regular, integral, and 
equivalued, (cf. 95.5) with valuation $s \in \mathbb{R}$. For any $t \in \mathbb{R}$ we associate to this data the affine Springer fiber $\mathcal{F}_{y}(t, u)$ of $\$ 4.1$. We need to show, for $t=0$, that it has a Hessenberg paving.

The conjugacy class of the torus $T$ is determined by the invariant $y(T) \in$ $H^{1}(F, W)$ of $\$ 5.2$. Without loss of generality, we may assume that $T$ is the torus constructed in $\$ 5.3$ with this invariant. Let $x \in \mathfrak{a}$ be the element constructed in the first paragraph of $\$ 5.4$ and the last paragraph of $\$ 5.5$. In $\$ 5.5$ it is verified that $u \in \mathfrak{g}_{x, s}$ and that its image $u^{\prime \prime} \in \mathfrak{g}$ is $G$-good. Therefore, for any $t \leq s$ the pair $(t, u)$ satisfies the hypotheses of 44.2 . Let us therefore apply the results of $\$ 4$.

The affine flag variety $\mathcal{F}_{y}$ admits a filtration by closed subschemes such that each successive difference is a single $G_{x}$ orbit. The induced filtration of the affine Springer fiber $\mathcal{F}_{y}(t, u)$ has successive differences isomorphic to

$$
S=\mathcal{F}_{c y}(t, u) \cap \mathbf{G}_{x} \cdot\left(\text { basepoint of } \mathcal{F}_{c y}\right)
$$

for various choices of $c$ in the normalizer of $A(F)$, cf. 4.3 By 44.4 the Moy-Prasad filtration (\$3) of $\mathfrak{g}$ determines a finite sequence of schemes

$$
S=S_{m} \rightarrow S_{m-1} \rightarrow \cdots \rightarrow S_{1}=S_{0+}
$$

with the property that

(1) $S_{0+}$ is a Hessenberg variety (cf. 84.5 ) and

(2) each $S_{j+1} \rightarrow S_{j}$ is an affine space bundle (cf. 4.7 ).

This concludes the proof that the affine Springer fiber has a Hessenberg paving. The modifications that are needed for the proof of Theorem 1.2 are described in Section 5.6 .

7.2. Proof of Corollary 1.3, It suffices to show the following: If an ind-scheme $X$ over a finite field $\mathbf{F}_{q}$ admits a Hessenberg paving $X_{0} \subset X_{1} \subset \cdots$ defined over $\mathbf{F}_{q}$, then the étale homology $H_{*}\left(X, \mathbf{Q}_{l}\right)$ (for $l$ prime to $q$ ) is pure, that is, all the eigenvalues of the geometric Frobenius on $H_{i}\left(X, \mathbf{Q}_{l}\right)$ (with $l$ prime to $q$ ) have complex absolute value $q^{i / 2}$.

For each $j$ the difference $X_{j} \backslash X_{j-1}$ is an iterated affine space bundle of some rank, $d_{j}$, over a Hessenberg variety $S_{j}$. The Thom isomorphism $H_{i}\left(S_{j}, \mathbf{Q}_{l}\right) \cong$ $H_{i+2 d_{j}}\left(X_{j}, X_{j-1}, \mathbf{Q}_{l}\right)$ takes Frobenius eigenvalues $\lambda$ with $|\lambda|=q^{i / 2}$ to eigenvalues $\lambda^{\prime}$ with $\left|\lambda^{\prime}\right|=q^{\left(i+2 d_{j}\right) / 2}$. It follows by induction on $j$ that $H_{*}\left(X_{j}, \mathbf{Q}_{l}\right)$ is pure and that the connecting homomorphism $H_{i}\left(X_{j}, X_{j-1}, \mathbf{Q}_{l}\right) \rightarrow H_{i-1}\left(X_{j-1}, \mathbf{Q}_{l}\right)$ vanishes. The homology $H_{*}\left(X, \mathbf{Q}_{l}\right)$ is the direct limit of the $H_{*}\left(X_{j}, \mathbf{Q}_{l}\right)$ so it is also pure. This completes the proof of Corollary 1.3.

We remark that the notion of a Hessenberg paving of an ind-scheme $X$ over $\mathbf{F}_{q}$ may be weakened slightly by removing the requirement that the sub-schemes $X_{j}$ be complete. In this case the preceding purity statement will remain valid provided we replace the étale homology $H_{*}\left(X, \mathbf{Q}_{l}\right)$ by the direct limit

$$
\lim _{j \rightarrow \infty} H_{*}^{B M}\left(X_{j}, \mathbf{Q}_{l}\right)
$$

of the Borel-Moore étale homology of the $X_{j}$. 


\section{REFERENCES}

[Ad198] J. Adler, Refined anisotropic K-types and supercuspidal representations, Pacific J. Math. 185 (1998), 1-32. MR 1653184 (2000f:22019)

[BEG] Y. Berest, P. Etingof, and V. Ginzburg, Finite dimensional representations of rational Cherednik algebras, Int. Math. Res. Not. 2003, no. 19, 1053-1088. MR1961261 (2004h:16027)

[Bez96] R. Bezrukavnikov, The dimension of the fixed point set on affine flag manifolds, Math. Res. Lett. 3 (1996), 185-189. MR1386839(97e:17024)

[Bor69] A. Borel, Linear algebraic groups, Benjamin, New York, 1969. MR0251042 (40 \#4273)

[CLP88] C. De Concini, G. Lusztig, and C. Procesi, Homology of the zero-set of a nilpotent vector field on a flag manifold, J. Amer. Math. Soc. 1 (1988), 15-34. MR0924700 (89f:14052)

[Fan96] C. Kenneth Fan, Euler characteristic of certain affine flag varieties, Transform. Groups 1 (1996), 35-39. MR1390748 (97b:17023)

[GKM98] M. Goresky, R. Kottwitz, and R. MacPherson, Equivariant cohomology, Koszul duality, and the localization theorem, Inv. Math. 131 (1998), 25-83. MR.1489894 (99c:55009)

[GKM04] M. Goresky, R. Kottwitz, and R. MacPherson, Homology of affine Springer fibers in the unramified case, Duke Math. J. 121 (2004), 509-561. MR2040285 (2005a:14068)

[KL88] D. Kazhdan and G. Lusztig, Fixed point varieties on affine flag manifolds, Israel J. Math. 62 (1988), 129-168. MR0947819 (89m:14025)

[Kos59] B. Kostant, The principal three-dimensional subgroup and the Betti numbers of a complex simple Lie group, Amer. J. Math. 81 (1959), 973-1032. MR0114875 (22 \#5693)

[L83] R.P. Langlands, Les débuts d'une formule des traces stables, Publ. Math. Univ. Paris VII 13, Université de Paris VII, U.E.R. de Mathemétiques, Paris, 1983. MR0697567 (85d:11058)

[Lau] G. Laumon, Sur le lemme fondamental pour les groupes unitaires, math.AG/0212245.

[LW] G. Laumon and J.-L. Waldspurger, Sur le lemme fondamental pour les groupes unitaires: le cas totalement ramifié et homogène, math.AG/9901114.

[Luc03] V. Lucarelli, Affine pavings for affine Springer fibers for split elements in PGL(3), math.RT/0309132

[LS91] G. Lusztig and J. M. Smelt, Fixed point varieties on the space of lattices, Bull. London Math. Soc. 23 (1991), 213-218. MR.1123328 (93e:14065)

[MP94] A. Moy and G. Prasad, Unrefined minimal K-types for p-adic groups, Invent. Math. 116 (1994), 393-408. MR.1253198 (95f:22023)

[MPS92] F. De Mari, C. Procesi, and M. A. Shayman, Hessenberg varieties, Trans. Amer. Math. Soc. 332 (1992), 529-534. MR1043857 (92j:14060)

[Sag00] D. Sage, The geometry of fixed point varieties on affine flag manifolds, Trans. Amer. Math. Soc. 352 (2000), 2087-2119. MR1491876 (2000j:14074)

[Ser68] J-P. Serre, Corps locaux, Hermann, Paris, 1968. MR0354618 (50 \#7096)

[Som97] E. Sommers, A family of affine Weyl group representations, Transform. Groups 2 (1997), 375-390. MR1486037 (98m:20016)

[SS70] T. Springer and R. Steinberg, Conjugacy classes, Seminar in Algebraic Groups and Related Finite Groups, Lecture Notes in Math., 131, Springer, 1970, pp. 167-266. MR.0268192(42 \#3091)

[Vas] E. Vasserot, Induced and simple modules of double affine Hecke algebras, Duke Math. J. 126 (2005), 251-323. MR2115259

[Yu01] Jiu-Kang Yu, Construction of tame supercuspidal representations, J. Amer. Math. Soc. 14 (2001), 579-622. MR.1824988 (2002f:22033)

School of Mathematics, Institute for Advanced Study, Princeton, New Jersey 08540

Department of Mathematics, University of Chicago, 5734 University Ave., Chicago, ILLINOIS 60637

School of Mathematics, Institute for Advanced Study, Princeton, New Jersey 08540 